\title{
Erratum to: Oral rehabilitation with dental implants in irradiated patients: a meta-analysis on implant survival
}

\author{
E. Schiegnitz ${ }^{1}$ B. Al-Nawas ${ }^{1}$ P. W. Kämmerer ${ }^{1,2} \cdot$ K. A. Grötz ${ }^{3}$
}

Published online: 18 June 2015

(C) Springer-Verlag Berlin Heidelberg 2015

\section{Erratum to: Clin Oral Invest 18 (3): 687-698 DOI 10.1007/s00784-013-1134-9}

The following amendments should be considered in the published article: Oral rehabilitation with dental implants in irradiated patients: a meta-analysis on implant survival. Schiegnitz E, Al-Nawas B, Kämmerer PW, Grötz KA. Clin Oral Investig. 2014 Apr;18(3):687-98. doi: 10.1007/s00784013-1134-9. In table 1, the below mentioned points concerning jaw region and time of implant placement should be corrected.

\section{Corrected version}

In table 1, in the studies of Schoen et al. (18), Schepers et al. (37) and Korfage et al. (16) mandibular implants were inserted pre-irradiation. In the studies of Klein et al. (17), Betz et al. (55) and Watzinger et al. (19) implants were inserted postirradiation.

The online version of the original article can be found at http://dx.doi.org/ 10.1007/s00784-013-1134-9.

\section{E. Schiegnitz}

eik.schiegnitz@unimedizin-mainz.de

1 Department of Oral and Maxillofacial Surgery, Plastic Surgery, University Medical Centre of the Johannes Gutenberg-University Mainz, Augustusplatz 2, 55131 Mainz, Germany

2 Harvard Medical School, Boston, MA, USA

3 Department of Oral and Maxillofacial Surgery, Dr. Horst Schmidt Clinic Wiesbaden, Wiesbaden, Germany
In our discussion section the passage "in this period, six studies regarding implant survival in the irradiated jaw compared to the non-irradiated jaw were published [15-18, 21, 25]. All of them showed no significant difference in implant survival between the irradiated and the non- irradiated jaw" should be corrected.

\section{Corrected version}

In this period, six studies regarding implant survival in the irradiated jaw compared to the non-irradiated jaw were published [15-18, 21, 25]. Four of them showed no significant difference in implant survival between the irradiated and the non-irradiated jaw. Korfage et al. (16) reported higher failure rates in irradiated bone. Linsen et al. (21) indicated lower implant survival in irradiated bone only after adjustment of data for implant loss due to resection of recurrent tumour. In Figure 2, the following points concerning the ratios implant failures to total implant numbers and mean follow-up should be corrected.

Corrected version:

After contacting the authors of the study of Klein et al. (17) the following ratios regarding implant failures to total implant numbers were obtained: non-irradiated native bone $2 / 23$ and irradiated native bone $1 / 39$. This resulted in a non statistically significant difference in implant survival between nonirradiated native bone and irradiated native bone (odds ratio [OR], 3.16; confidence interval [CI], 0.99-10.11) as described in our study. Excluding the study of Linsen et al. (21) because of mean follow-up of 42 months, the meta-analysis will still have the same result ([OR], 2.83; [CI], 0.80-9.99) and we would draw the same conclusions out of our study. 